\title{
A two-year follow-up Study: Validity of bioelectrical impedance analysis to detect alteration in appendicular lean mass in Arab men
}

\section{Shaea A. Alkahtani}

Department of Exercise Physiology, College of Sport Sciences and Physical Activity, King Saud University, Riyadh, Saudi Arabia

\section{Abstract:}

The aim of current study was to determine the validity of bioelectrical impedance analysis (BIA) to detect a 2-year follow-up in body composition compared with dual-energy $X$ ray absorptiometry (DXA). A follow-up repeated measure design was implemented. 51 healthy men with an average age $33.8 \pm 12.2$ years, and average Body Mass Index (BMI) of $29.1 \pm 5.1$ $\mathrm{kg} / \mathrm{m} 2$ participated in the current study. The average appendicular lean mass (ALM) divided by height squared (ALM/ht2) and pre-sarcopenia as 1 SD below the average value based on specific reference values of young Saudi men were used. The reference value for DXA was 7.74 $\mathrm{kg} / \mathrm{m} 2$ and for Tanita was $8.68 \mathrm{~kg} / \mathrm{m} 2$. Results showed no significant differences in body composition between baseline and follow up measures using DXA and Tanita $(P>0.05)$. Tanita has a sensitivity of $66.7 \%$ and specificity of $69.6 \%$ compared to DXA at baseline and had a sensitivity of $100.0 \%$ and specificity of $86.4 \%$ when compared to DXA. Bland-Altman analysis showed that the mean difference between the measure of Tanita at baseline and follow up was 0.17 with upper and lower limit for agreement 1.82 and -2.17 respectively, and the comparison for DXA at baseline and follow up showed mean difference-0.04 with upper and lower limit for agreement 2.35 and -2.43 respectively. In conclusion, a short-term 2-year follow-up measures showed no differences in body composition including ALM. Tanita MC-980 has a strong level of sensitivity and specificity in the follow up measure in comparison with DXA.

\section{Introduction :}

The circumstances of sarcopenia occurrence are determined in a number of indices including bedridden, difficulty to rise from a chair and having a gait speed less than $1 \mathrm{~m} / \mathrm{s}$ (Fielding et al., 2011). Although these signs are likely to occur after the age of 70, deterioration of muscle quality and quantity through the loss of muscle mass and the decrease of muscle function can begin from the fourth decade (Cruz-Jentoft et al., 2010). For example, a 3-year follow up study found a decrease in muscle strength although muscle mass was preserved, which implies that muscle quality through decrease in muscle fiber types has more impact than the loss of muscle mass particularly among men (Goodpaster et al., 2006), and surprisingly the decline in strength was greater among participants with higher initial strength (Goodpaster et al., 2006). A 5-year follow-up study also found that the decrease in isokinetic leg muscle torque is more pronounced than the decrease in muscle crosssectional area, and weight gain did not prevent the decrease in muscle torque (Delmonico et al., 2009). A follow-up investigation of the alteration of body composition can help to examine the early onset of the decrease in muscle mass as a sign of pre-sarcopenia.

DXA is considered as the gold standard of body composition measures. However, bioelectrical impedance analysis is a widely used in clinic and research 
to assess body composition and has many advantages as a noninvasive portable instrument. The validity of BIA is affected by age groups including in children, adults, and elderlies. For example, BIA method understated fat mass in comparison with DXA among children and is highly affected by the progress of aging due to changes in lean mass, total body water and bone mass (Khalil, Mohktar, \& Ibrahim, 2014). While BIA has been recommended for follow-up clinical practice on a medium and long term basis, further research is suggested to examine its accuracy in the follow up measures in relation to specific health parameters and different population and ethnicities (Mialich, Sicchieri, \& Junior, 2014). The relationship between body mass index and adiposity is also race/ethnic dependent, such that using BMI as a phenotypic marker of adiposity across different populations may not be appropriate (Heymsfield, Peterson, Thomas, Heo, \& Schuna, 2016). It was accepted for measuring body composition over time among healthy individuals but was not recommended in individual patients (Buchholz, Bartok, \& Schoeller, 2004) and in large epidemiological studies (Dehghan \& Merchant, 2008). However, cross-sectional studies of the association between BIA and DXA showed inconsistent outcomes. For example, the estimation of percentage fat using BIA was significantly lower than DXA, and BIA tended to underestimated percentage fat in obese individuals and overestimated it in lean individuals (Sun et al., 2005). BIA underestimated fat mass and overestimated lean mass, and did not detect small changes in body composition after exercise training (Sillanpaa, Hakkinen, \& Hakkinen, 2013). On the other hand, a similar outcome between BIA and DXA was found in estimating lower limb skeletal muscle mass among obese and non-obese women, even with using different resistance values for the current of BIA machine (Salinari et al., 2003). BIA also accurately predict appendicular lean mass among healthy adults between 22 and 94 years (Kyle, Genton, Hans, \& Pichard, 2003), and BIA was not significantly different compared with MRI in estimating skeletal muscle mass (Chien, Huang, \& $\mathrm{Wu}, 2008)$. Thus, it is important to examine the sensitivity and specificity of BIA devices in comparison with DXA, and preferably overtime which has not been fully examined yet.

The sensitivity of a device is the proportion of actual positives which are correctly identified by such a device, and specificity is the proportion of true negatives which are correctly identified by the device as negative. Sensitivity, by definition, could be considered as a measure to quantify the ability to avoid false negatives and specificity could be used to quantify the ability to avoid false positives. We have recently found that Tanita MC-980 device has a moderate level of sensitivity (54\%) and a high level of specificity (98\%) in comparison with DXA (Alkahtani, 2017). The appropriate cut-off for Saudi population was determined in 
this study, but the consistency of BIA in follow up measures and its ability to detect small changes in body weight and lean mass alterations has not been confirmed yet. The aim of the current study is to examine the validity of BIA through the sensitivity and specificity of BIA device against DXA over two years follow up. This is important because changes in body composition compartment including loss of appendicular muscle mass are associated with the higher metabolic disorder and increased risk of mortality than obesity and sarcopenia alone (Wannamethee \& Atkins, 2015). Thus, follow up and longitudinal research of body composition can help to detect alterations in fat and muscle mass and the occurrence of pre-sarcopenia.

\section{Methods}

\section{Participants}

51 healthy men with an average age $33.8 \pm 12.2$ years, and average BMI of $29.1 \pm 5.1 \mathrm{~kg} / \mathrm{m}^{2}$ participated in the current study. The first participation was in February 2016, which was announced at King Saud University (KSU) and Riyadh City in Kingdome of Saudi Arabia, and 497 men participated in the study measures, with a few participants from other cities outside Riyadh. The second follow-up invitation was sent to the participants in February 2018, and 51 participants came and started the measures. 42 participants performed bioelectrical impedance analysis (BIA) measures using Tanita, and 35 participants performed dual-energy X-ray absorptiometry (DXA) measures, and 26 participants of them completed BIA and DXA measures. Participants lived freely in the period between the $1^{\text {st }}$ and $2^{\text {nd }}$ visits, and there was not any type of monitoring for participants in this period. All participants signed a consent form confirming their volunteer participation in prior to the first and second measures. The consent form included information of the exposure to radiation and maximal allowance for the patient, and a research assistant was available to answer questions.

\section{Study procedure}

A follow-up repeated measure design was implemented. The same instruments and procedure of anthropometry, handgrip strength and body composition measures including DXA and BIA were used in the first and second measures. The first and second measures were performed at Body Composition Laboratory at College of Sport Sciences and Physical Activity at KSU.

Body composition was measured using DXA machine (Lunar iDXA, GE Healthcare, USA). Quality Assurance Calibration was performed prior to tests. Participants' data were inserted, and participants were bedded on a supine position. Participants were required to remain motionless during the test, and output was printed at the end of the test.

Body composition was also measured using BIA from Tanita MC-980MA 
(Tanita Corporation, Tokyo, Japan), which is a multi-frequency monitor that is able to measure impedance at 5, 50, 250,500 and $1000 \mathrm{kHz}$ to make it possible to estimate extar0 and intra-cellular water. Participants were requested to stand on the scale bare feet and hold the handles of the machines, following visual instructions of the test procedure. Fat mass, lean mass and appendicular lean mass which is the sum of the arm and leg lean mass were recorded from the output sheet for each participant.

The average ALM/ht ${ }^{2}$ and pre-sarcopenia as $1 \mathrm{SD}$ below the average value were determined based on specific reference values of young Saudi men (Alkahtani, 2017), such that the reference value for DXA was $7.74 \mathrm{~kg} / \mathrm{m}^{2}$ and for Tanita was $8.68 \mathrm{~kg} / \mathrm{m}^{2}$.

\section{Statistical analysis}

Data were analyzed using SPSS (version 20 Chicago, IL, USA). Kolmogorov-Smirnov test was used to check the normality of all continuous variables. Data were presented as mean \pm standard error (SEM). Paired samples ttest and Wilcoxon signed-rank test were used to identify the differences between baseline and follow-up measurements for Gaussian and non-Gaussian distributed variables, respectively. The agreement between the two used devices (DXA and Tanita) was assessed using Cohen's Kappa values based on both baseline and follow-up measurements. Bland-Altman plot was used to show the agreement between measures at baseline and follow-up. Sensitivity and specificity of Tanita as compared to DXA at baseline and 2-years follow-up were calculated using the following formula: Sensitivity $=\mathrm{TP} /(\mathrm{TP}+\mathrm{FN})$, and specificity $=\mathrm{TN} /(\mathrm{TN}+\mathrm{FP})$, where TP means true positive; FP means false positive FN means false negative and TN means true negative. A significance level of 0.05 was adopted for all the different tests.

\section{Results}

Table 1 showed the comparisons between baseline and follow-up in anthropometric characteristics of the study participants.

\section{Table 1.}

Comparisons between baseline and follow-up anthropometric characteristics

\begin{tabular}{cccc}
\multicolumn{4}{c}{ of the study participants $(\mathrm{N}=51)$, data shown as Mean \pm SEM. } \\
\hline Variables & Baseline & Follow-up & $P$ value \\
\hline Weight $(\mathrm{kg})$ & $83.49 \pm 2.24$ & $85.85 \pm 2.07$ & 0.001 \\
BMI $\left(\mathrm{kg} / \mathrm{m}^{2}\right)$ & $28.49 \pm 0.73$ & $29.23 \pm 0.66$ & 0.001 \\
WC $(\mathrm{cm})$ & $92.51 \pm 1.82$ & $96.57 \pm 2.53$ & 0.001 \\
HC $(\mathrm{cm})$ & $104.66 \pm 1.49$ & $107.05 \pm 1.59$ & 0.016 \\
WHR & $0.88 \pm 0.01$ & $0.90 \pm 0.02$ & 0.023 \\
\hline
\end{tabular}

SEM: standard error of mean, BMI: body mass index, WC: waist circumference, HC: hip circumference, WHR: waist to hip ratio. 
Table 2 showed differences in body composition measures using DXA and Tanita at baseline and follow up, which were not significant $(\mathrm{P}>0.05)$.

Table 2.

Comparisons between DXA and Tanita measures at baseline and follow-up, data shown as Mean \pm SEM..

\begin{tabular}{|c|c|c|c|c|c|c|c|c|}
\hline \multirow[b]{2}{*}{ Variables } & \multicolumn{4}{|c|}{ DXA $(n=35)$} & \multicolumn{4}{|c|}{ Tanita $(\mathrm{n}=42)$} \\
\hline & $\begin{array}{c}\text { Baseline } \\
(\text { Mean } \pm \\
\text { SEM) }\end{array}$ & $\begin{array}{c}\text { Follow-up } \\
\text { (Mean } \pm \\
\text { SEM) }\end{array}$ & $\begin{array}{c}P \\
\text { value }\end{array}$ & $\begin{array}{l}\text { Effect } \\
\text { size }\end{array}$ & $\begin{array}{c}\text { Baseline } \\
\text { (Mean } \pm \\
\text { SEM) }\end{array}$ & $\begin{array}{c}\text { Follow-up } \\
\text { (Mean } \pm \\
\text { SEM) }\end{array}$ & $\begin{array}{c}P \\
\text { value }\end{array}$ & $\begin{array}{l}\text { Effect } \\
\text { size }\end{array}$ \\
\hline Body fat $\%$ & $29.34 \pm 1.57$ & $29.41 \pm 1.46$ & 0.95 & 0.01 & $25.25 \pm 1.20$ & $25.41 \pm 1.13$ & 0.91 & 0.02 \\
\hline Lean $(\mathrm{kg})$ & $53.75 \pm 0.93$ & $54.39 \pm 1.14$ & 0.57 & 0.10 & $57.78 \pm 1.07$ & $58.56 \pm 1.07$ & 0.45 & 0.12 \\
\hline $\begin{array}{c}\text { Lean } / \mathrm{Ht}^{2} \\
\left(\mathrm{~kg} / \mathrm{m}^{2}\right)\end{array}$ & $18.48 \pm 0.34$ & $18.58 \pm 0.30$ & 0.80 & 0.04 & $19.81 \pm 0.33$ & $19.99 \pm 0.31$ & 0.63 & 0.08 \\
\hline $\operatorname{ALM}(\mathrm{kg})$ & $26.22 \pm 0.57$ & $26.47 \pm 0.71$ & 0.68 & 0.07 & $27.52 \pm 0.60$ & $27.68 \pm 0.61$ & 0.77 & 0.04 \\
\hline ALM/BMI & $0.99 \pm 0.03$ & $0.96 \pm 0.02$ & 0.24 & 0.20 & $0.99 \pm 0.03$ & $0.96 \pm 0.01$ & 0.13 & 0.24 \\
\hline $\begin{array}{c}\mathrm{ALM} / \mathrm{Ht}^{2} \\
\left(\mathrm{~kg} / \mathrm{m}^{2}\right)\end{array}$ & $9.01 \pm 0.19$ & $9.04 \pm 0.20$ & 0.88 & 0.02 & $9.43 \pm 0.18$ & $9.45 \pm 0.19$ & 0.90 & 0.02 \\
\hline
\end{tabular}

SEM: standard error of means; ALM: appendicular lean mass; $\mathrm{Ht}^{2}$ : height squared meter; BMI: body mass index;

Table 3 showed alteration in body composition using Tanita. While data seems to be similar at baseline and follow up measure, individual variations demonstrated a very large range in the differences between baseline and follow up among individuals. 8 participants gained $\geq 10 \mathrm{~kg}$ and 8 participants lost $\geq 10 \mathrm{~kg}$ in the two years follow up period.

Table 3.

Individual variations in body composition at baseline and follow up measures using Tanita $(\mathrm{n}=42)$, data shown as Mean \pm SEM.

\begin{tabular}{|c|c|c|c|c|c|c|c|c|c|}
\hline \multirow[b]{2}{*}{ Variable } & \multicolumn{3}{|c|}{ Baseline } & \multicolumn{3}{|c|}{ Follow up } & \multicolumn{3}{|c|}{ Difference } \\
\hline & $\begin{array}{l}\text { Mean } \\
\pm \text { SEM }\end{array}$ & Range & $\mathrm{CV} \%$ & $\begin{array}{l}\text { Mean } \\
\pm \text { SEM }\end{array}$ & Range & $\mathrm{CV} \%$ & $\begin{array}{l}\text { Mean } \\
\pm \text { SEM }\end{array}$ & Range & CV \% \\
\hline $\begin{array}{c}\text { Body } \\
\text { weight }(\mathrm{kg})\end{array}$ & $\begin{array}{l}82.19 \\
\pm 2.78\end{array}$ & $\begin{array}{l}(51.80- \\
132.50)\end{array}$ & 21.69 & $\begin{array}{l}83.89 \\
\pm 2.56\end{array}$ & $\begin{array}{l}(54.70- \\
123.50)\end{array}$ & 19.57 & $\begin{array}{c}1.70 \\
\pm 2.38\end{array}$ & $\begin{array}{c}(-29.10- \\
46.80)\end{array}$ & 898.63 \\
\hline FM (kg) & $\begin{array}{l}21.78 \\
\pm 1.78\end{array}$ & $\begin{array}{l}(7.80- \\
55.50)\end{array}$ & 52.53 & $\begin{array}{l}22.31 \\
\pm 1.52\end{array}$ & $\begin{array}{l}(9.90- \\
47.30)\end{array}$ & 43.75 & $\begin{array}{c}0.53 \\
\pm 1.78\end{array}$ & $\begin{array}{c}(-38.00- \\
33.00)\end{array}$ & 2145.84 \\
\hline FFM (kg) & $\begin{array}{l}60.42 \\
\pm 1.17\end{array}$ & $\begin{array}{c}(43.80- \\
77.00)\end{array}$ & 12.42 & $\begin{array}{l}61.03 \\
\pm 1.18\end{array}$ & $\begin{array}{c}(44.50- \\
76.20)\end{array}$ & 12.41 & $\begin{array}{c}0.61 \\
\pm 1.10\end{array}$ & $\begin{array}{c}(-14.60- \\
14.50)\end{array}$ & 1148.85 \\
\hline TBW(kg) & $\begin{array}{l}43.54 \\
\pm 0.77\end{array}$ & $\begin{array}{c}(33.00- \\
56.60)\end{array}$ & 11.39 & $\begin{array}{l}43.80 \\
\pm 0.81 \\
\end{array}$ & $\begin{array}{c}(33.80- \\
55.30)\end{array}$ & 11.83 & $\begin{array}{c}0.26 \\
\pm 0.73 \\
\end{array}$ & $\begin{array}{l}(-8.50- \\
11.60)\end{array}$ & 1824.67 \\
\hline
\end{tabular}

SEM: standard error of means; CV: coefficient of variations; FM: fat mass; FFM: fat free mass; TBW: total body water.

Sensitivity and specificity at baseline analysis showed that Tanita has a sensitivity of $66.7 \%$ (as Tanita found 2 participants were positive out of 3 were identified by DXA) and specificity of $69.6 \%$ (as Tanita found 16 participants were negative out of 23 were identified by 
DXA) compared to DXA. Results of the follow-up analysis showed a sensitivity of $100.0 \%$ (as Tanita found 4 participants were negative out of 4 were identified by DXA) and specificity of $86.4 \%$ (as Tanita found 19 participants were negative out of 22 identified by DXA) as compared to DXA, (Table 4).

\section{Table 4.}

Sensitivity and specificity of Tanita compared to DXA in measuring ALM at baseline and Follow-up ( $\mathrm{n}=26)$.

Measures of appendicular lean mass Baseline Follow up

\begin{tabular}{|c|c|c|c|c|c|c|c|c|}
\hline \multirow{2}{*}{\multicolumn{3}{|c|}{ (ALM) }} & \multicolumn{2}{|c|}{ DXA } & \multirow{2}{*}{ Total } & \multicolumn{2}{|c|}{ DXA } & \multirow{2}{*}{ Total } \\
\hline & & & Negative & Positive & & Negative & Positive & \\
\hline \multirow{6}{*}{ Tanita } & & Count & 16 & 1 & 17 & 19 & 0 & 19 \\
\hline & Negative & $\%$ within Tanita & $94.1 \%$ & $5.9 \%$ & $100.0 \%$ & $100.0 \%$ & $0.0 \%$ & $100.0 \%$ \\
\hline & & \% within DXA & $69.6 \%$ & $33.3 \%$ & $65.4 \%$ & $86.4 \%$ & $0.0 \%$ & $73.1 \%$ \\
\hline & \multirow{3}{*}{ Positive } & Count & 7 & 2 & 9 & 3 & 4 & 7 \\
\hline & & $\%$ within Tanita & $77.8 \%$ & $22.2 \%$ & $100.0 \%$ & $42.9 \%$ & $57.1 \%$ & $100.0 \%$ \\
\hline & & $\%$ within DXA & $30.4 \%$ & $66.7 \%$ & $34.6 \%$ & $13.6 \%$ & $100.0 \%$ & $26.9 \%$ \\
\hline & otal & & 23 & 3 & 26 & 22 & 4 & 26 \\
\hline
\end{tabular}

Baseline: Sensitivity $=\mathrm{TP} /(\mathrm{TP}+\mathrm{FN})=2 /(2+1)=0.66$; Specificity $=\mathrm{TN} /(\mathrm{TN}+\mathrm{FP})=$ $16 /(16+7)=0.69$

Follow up: Sensitivity $=\mathrm{TP} /(\mathrm{TP}+\mathrm{FN})=4 /(4+0)=1 ;$ Specificity $=\mathrm{TN} /(\mathrm{TN}+\mathrm{FP})=$ $19 /(19+3)=0.86$

TP: true positive; FP: false positive; FN: false negative; TN: true negative.

Bland-Altman analysis showed that the difference in $\mathrm{ALM} / \mathrm{Ht}^{2}$ between mean DXA and Tanita was $-0.25 \mathrm{~kg} / \mathrm{m}^{2}$ and $-0.38 \mathrm{~kg} / \mathrm{m}^{2}$ at baseline and follow up respectively, with upper and lower limit for agreement of 0.99 and $-1.49 \mathrm{~kg} / \mathrm{m}^{2}$ at baseline and 0.90 and $-1.66 \mathrm{~kg} / \mathrm{m}^{2}$ at follow up (Figure 1).

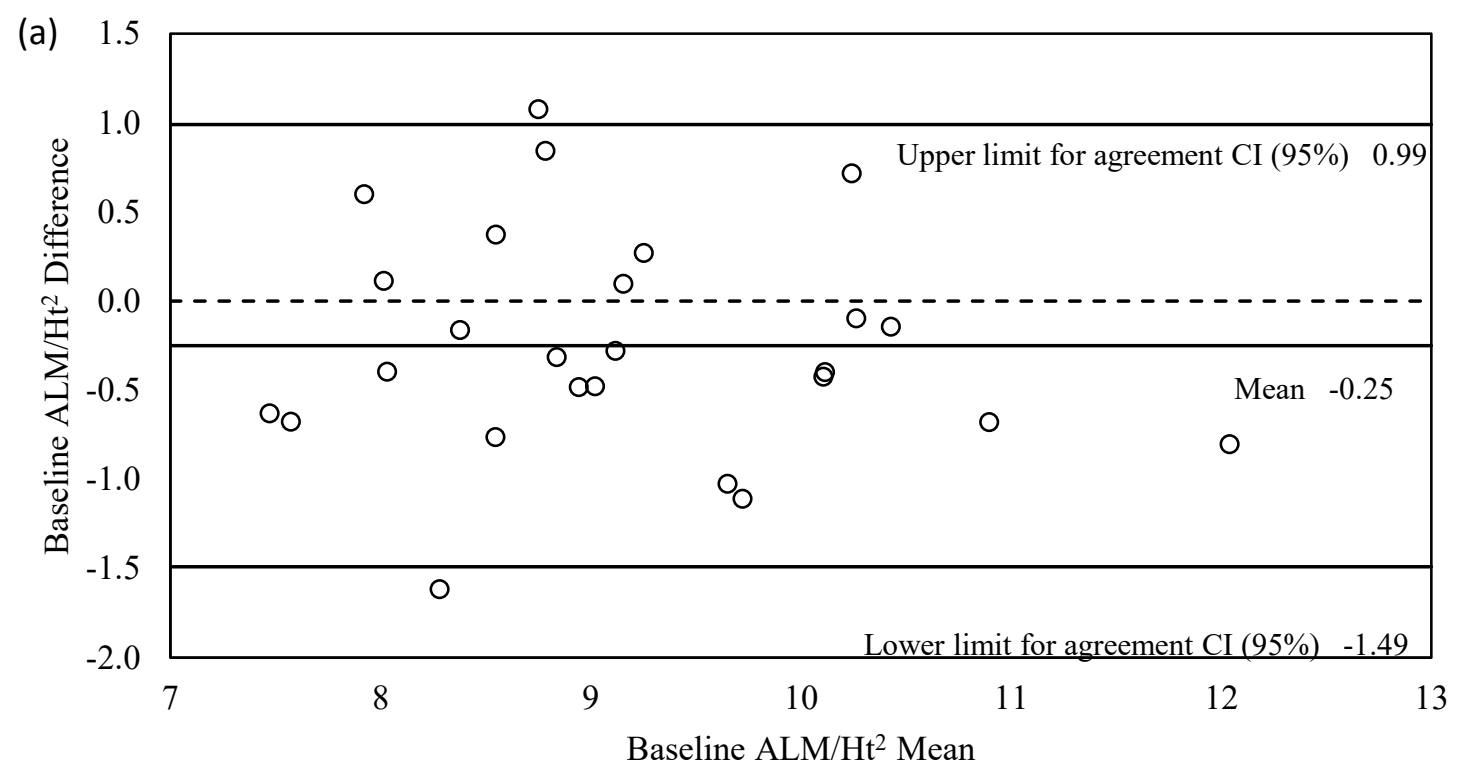




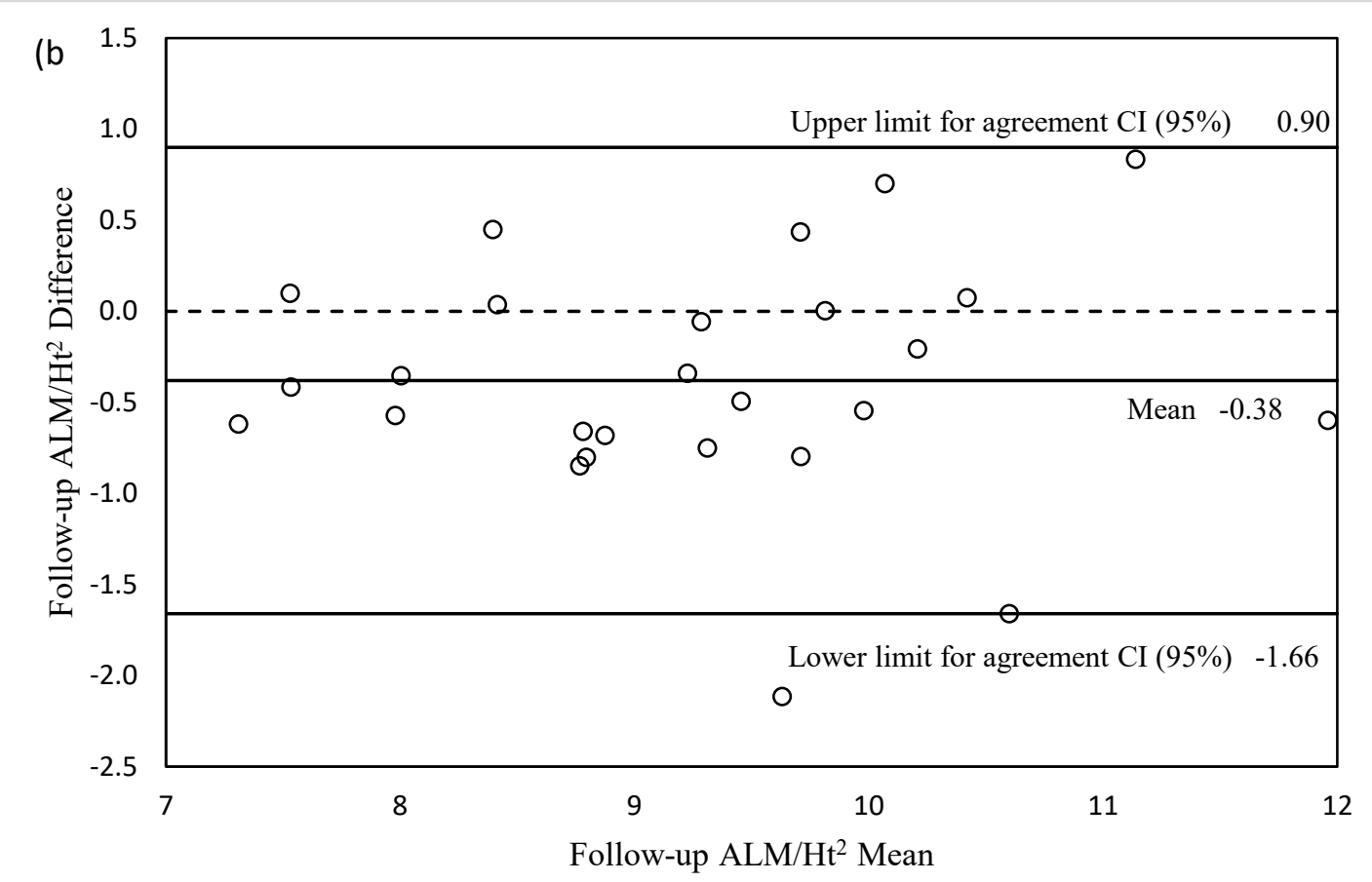

Figure 1.

Bland Altman plot showing the differences in ALM/ $\mathrm{Ht}^{2}$ between DXA and TANITA measurements at baseline (a) and Follow-up (b).

The comparison between the measure of Tanita at baseline and follow up showed mean difference $-0.17 \mathrm{~kg} / \mathrm{m}^{2}$ with upper and lower limit for agreement 1.82 and $-2.17 \mathrm{~kg} / \mathrm{m}^{2}$, and the comparison for DXA at baseline and follow up showed mean difference -0.04 with upper and lower limit for agreement 2.35 and -2.43 $\mathrm{kg} / \mathrm{m}^{2}$ (Figure 2).

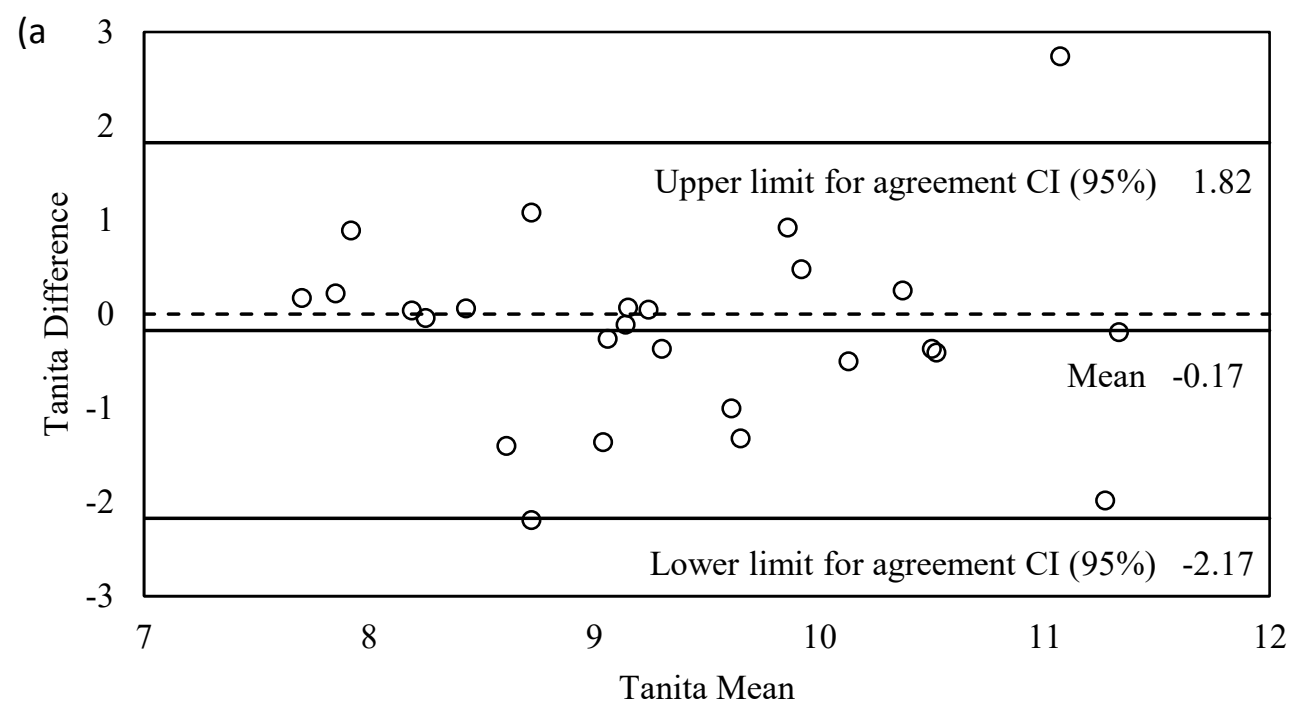




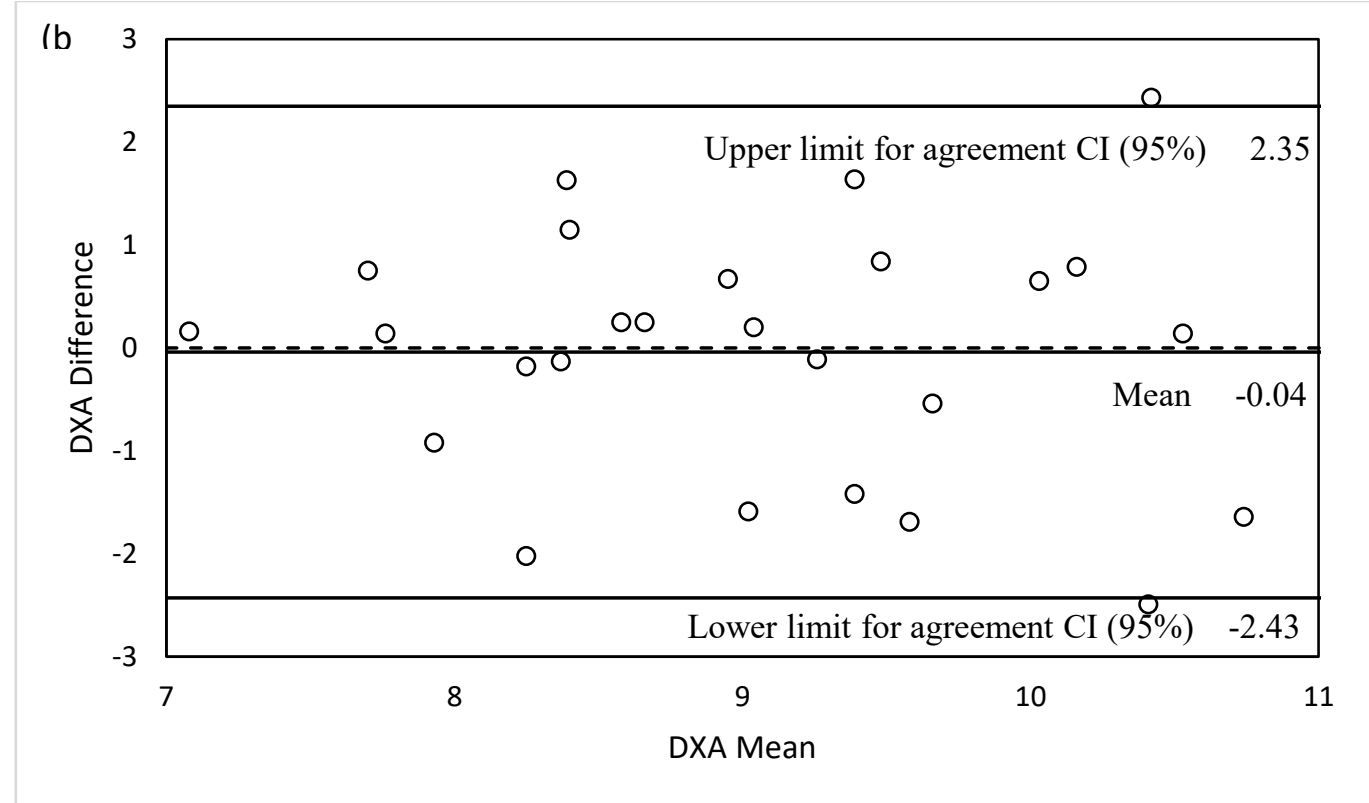

Figure 2.

Bland Altman plot demonstrating the difference between baseline and follow-up in appendicular lean mass/height squared meter $\left(\mathrm{ALM} / \mathrm{Ht}^{2}\right)$ using Tanita (a) and DXA (b), $\mathrm{n}=26$.

\section{Discussion}

Our results showed very good sensitivity (from $66.7 \%$ at baseline into $100.0 \%$ at follow-up) and specificity (from $69.6 \%$ at baseline into $86.4 \%$ at followup) of Tanita measure. It should be noted that data of 26 participants were used in this analysis in the current study, each participant account for $3.85 \%$ of the total of 26 participants. In such case, for example, at baseline Tanita found 2 participants were positive out of 3 were identified by DXA, in which, one participant account for $33.33 \%$. If there were a larger sample size, this percentage would be changed for all sensitivity and specificity measurements of Tanita compared to DXA both at baseline and follow-up. Thus, the current study showed a strong sensitivity and specificity level of Tanita in comparison to DXA in the follow-up measure although the small sample size, compared to some previous studies. For example, malnutrition was assessed over 2 years using a single-frequency BIA in patients receiving hemodialysis. This study found that sensitivity based on the cutoff points of resistance/height and impedance/height together ranged from $73 \%$ to $89 \%$ and specificity ranged from $49 \%$ to $50 \%$ in men, and sensitivity ranged from $58 \%$ to $80 \%$ and specificity from $48 \%$ to $55 \%$ in women. Based on the analysis of sensitivity and specificity, the author concluded that BIA parameters demonstrated low to moderate accuracy in men and low accuracy in women for the diagnosis of malnutrition (da Silva, Hauschild, Moreno, Bastos, \& Wazlawik, 2018). The 
sensitivity, specificity, and positive and negative predictive values were calculated for four BIA devices in comparison with DXA in the assessment of adolescents. The tetrapolar BIA device equipped with eight tactile electrodes showed more sensitivity and results that were closer to those obtained by DXA and is capable of predicting alterations in adolescents' body composition (Goncalves, Faria, Franceschini Sdo, \& Priore, 2013).

Bland Altman analysis showed no systematic bias in the data between DXA and Tanita at baseline and follow up measures although small underestimation of Tanita in the mean difference of ALM $\left(-.25 \mathrm{~kg} / \mathrm{m}^{2}\right.$ and $\left.-0.38 \mathrm{~kg} / \mathrm{m}^{2}\right)$ at the baseline and follow up. All individuals were within limits of the agreement except 1 to 2 participants. There is a difficulty to compare the current agreement between DXA and Tanita MC-980 with previous studies that employed different populations and different devices because most available studies used lean mass in $\mathrm{kg}$ rather than using the index of sarcopenia which is appendicular lean mass against height squared meter. For example, the latest model of multi-frequency Inbody 720, which employed frequency current up to $500 \mathrm{kHz}$ unlike the current Tanita which employed up to $1000 \mathrm{kHz}$, showed systematic bias among older Japanese with overestimation of the appendicular fat mass (bias $=-0.3 \mathrm{~kg}$ ) and underestimation of appendicular lean mass (bias $=1.5 \mathrm{~kg}$ ), and this study showed the opposite trend of the proportional bias of whole body fat mass compared with appendicular fat mass and lean mass and whole body lean mass compared with appendicular lean mass (Kim \& Kim, 2013). Another study on Chinese elderly using Inbody 720 showed that the mean difference between DXA and Inbody 720 in appendicular lean mass was $-0.99 \mathrm{~kg}$ and limits of agreement were -3.05 to $1.06 \mathrm{~kg}$ in men, and the mean difference was $-0.49 \mathrm{~kg}$ and limits of agreement were -2.40 to $1.41 \mathrm{~kg}$ in women (Wang et al., 2016). Moreover, our current repeated follow up measure of the same device of either DXA or Tanita showed very minor difference of means (-0.04 and $-0.17 \mathrm{~kg} / \mathrm{m}^{2}$ for DXA and Tanita respectively), but wider limits of agreement compared with the narrow limits of agreement between DXA and Tanita that have been taken at the same time on baseline or follow up. This could be attributed to the large variability in the body weight from baseline to follow up, which was greater than $\pm 10 \mathrm{~kg}$ among 16 participants. In conclusion, it should be noted that Tanita monitor uses patented equations based on hundred-thousands of measures worldwide (Tanita's exclusive equations), including additional information such as gender, age, and height alongside weight and impedance data to the algorithm. The current model of Tanita uses multi-frequency currents up to $1000 \mathrm{kHz}$ to accurately estimate extra-cellular water, intra-cellular water, and total body water, which is higher than suggested level $(300 \mathrm{kHz})$ to reach an acceptable level of accuracy (Pietrobelli et al., 1998). Thus, the current limits of agreements between 
DXA and Tanita is narrower than other devices and equations that were larger than $\pm 2 \mathrm{~kg}$ (Reiter et al., 2019), and also overcome the systematic bias that was reported in different devices including Tanita MC-180 which uses only 4 different frequencies up to $500 \mathrm{kHz}$ (Ellegard, Aldenbratt, Svensson, \& Lindberg, 2019). Our current preliminary outcomes also suggested better sensitivity and specificity of Tanita-MC-980 to detect the same level of changes in body composition compared to other devices in similar studies.

The strength of this study included that it has been conducted in one laboratory using the same devices and the same research assistants. Future followup studies from our laboratory and other laboratories on Arabs populations should consider the consistency of devices and preferably recruit the same research assistants or assistants with the same training level. The limitation of the study is the small sample size in the second measures, which could be attributed to the unfamiliarity of participants with the concept of follow up studies. The current model of Tanita showed better agreement with DXA than other BIA devices, but it is the latest and the most expensive model of Tanita which is not usually used by clinics and fitness clubs. Future studies should include these empirically used models of Tanita. It should be noted that the sensitivity and specificity of a BIA device can be different for the whole body compared to segmental parts such as arms and legs. Methodological studies are highly needed, including the calculation of the phase angle to address the accuracy of these differences.

Future studies should consider several factors such as obesity degree and gender difference which may affect the level of sensitivity and specificity of BIA devices. For example, the ability of tetrapolar BIA to detect excess weight in adolescents was examined, and sensitivity and specificity were high, ranging from $83 \%$ to $95 \%$ for girls and from $92 \%$ to $98 \%$ for boys, suggesting a slightly higher performance for the male gender (Neves et al., 2015). Although Tanita BF-689 demonstrated excellent test-retest reliability, moderately strong absolute agreement with DXA, it showed different sensitivity and specificity among children based on body fat showing high specificity for overweight and obese classification (Kabiri, Hernandez, \& Mitchell, 2015). Moreover, follow up measures of anthropometry in parallel with BIA is empirically important to address the accuracy of it. For example, the ability of BMI to reflect low percent body fat was evaluated, and moderate sensitivity (58\%) and high specificity (93\%) for BMI $<18.5$ were found, and based on this analysis the data suggest that BMI was not a good predictor of low \%BF (Gartner et al., 2000). There were different values of sensitivity and specificity of the percentiles of BMI, which sensitivity and specificity were 0.39 and 0.99 for the 95 th percentile of BMI, and 0.65 and 0.95 for the 85 th percentile of BMI. The study concluded that BMI percentiles have a high specificity but a 
low sensitivity in detecting excess adiposity in children (Bedogni et al., 2003). A systematic review and meta-analysis demonstrated the ability of BMI cutoff to detect body adiposity, and found that commonly used BMI cutoff values to diagnose obesity have high specificity $(90 \%)$ but low sensitivity $(50 \%)$ to identify adiposity as they fail to identify half of the people with excess BF\% (Okorodudu et al., 2010).

In conclusion, a short-term 2-year follow up measures showed no differences in body composition including ALM. Tanita MC-980 has a strong level of sensitivity and specificity in the follow-up measure in comparison with DXA.

\section{Declarations:}

Funding: This study received a grant from the Deanship of Scientific Research at King Saud University.

Acknowledgments: The author thanks all research assistants and participants. The authors also thank the Deanship of Scientific Research and RSSU at King Saud University for their technical support.

Ethical Approval: The study protocol was approved by the institutional review board (IRB) of King Saud University (IRB No. KSU-SE-18-11). Written informed consent was provided by all the participants.

Conflicts of Interest: The author declares no conflict of interest.

Availability of data and materials: All raw data presented in the manuscript are freely available to any scientist wishing to use them for non-commercial purposes, without breaching participants' confidentiality.

\section{References}

Alkahtani, S. A. (2017). A cross-sectional study on sarcopenia using different methods: reference values for healthy Saudi young men. BMC Musculoskeletal Disorders, 18(1), 119. doi:10.1186/s12891-017-1483-7

Bedogni, G., Iughetti, L., Ferrari, M., Malavolti, M., Poli, M., Bernasconi, S., \& Battistini, N. (2003). Sensitivity and specificity of body mass index and skinfold thicknesses in detecting excess adiposity in children aged 8-12 years. Annals of Human Biology, 30(2), 132-139. doi:10.1080/0301446021000033409

Buchholz, A. C., Bartok, C., \& Schoeller, D. A. (2004). The Validity of Bioelectrical Impedance Models in Clinical Populations. Nutrition in Clinical Practice, 19(5), 433-446. doi:doi:10.1177/0115426504019005433

Chien, M.-Y., Huang, T.-Y., \& Wu, Y.-T. (2008). Prevalence of Sarcopenia Estimated Using a Bioelectrical Impedance Analysis Prediction Equation in Community-Dwelling Elderly People in Taiwan. Journal of the American Geriatrics Society, 56(9), 1710-1715. doi:doi:10.1111/j.1532-5415.2008.01854.x

Cruz-Jentoft, A. J., Baeyens, J. P., Bauer, J. M., Boirie, Y., Cederholm, T., Landi, F., . . . European Working Group on Sarcopenia in Older, P. (2010). Sarcopenia: European consensus on definition and diagnosis: Report of the European Working Group on Sarcopenia in Older People. Age Ageing, 39(4), 412-423. doi:10.1093/ageing/afq034

da Silva, A. T., Hauschild, D. B., Moreno, Y. M. F., Bastos, J. L. D., \& Wazlawik, E. (2018). 
Diagnostic Accuracy of Bioelectrical Impedance Analysis Parameters for the Evaluation of Malnutrition in Patients Receiving Hemodialysis. Nutr Clin Pract, 33(6), 831-842. doi:10.1002/ncp.10098

Dehghan, M., \& Merchant, A. T. (2008). Is bioelectrical impedance accurate for use in large epidemiological studies? Nutrition Journal, 7(1), 26. doi:10.1186/1475-2891-7-26

Delmonico, M., Harris, T., Visser, M., Park, S., Conroy, M., Velasquez-Mieyer, P., . . . Goodpaster, B. (2009). Longitudinal study of muscle strength, quality, and adipose tissue infiltration. The American Journal of Clinical Nutrition, 90(6), 1579-1585. doi:10.3945/ajen.2009.28047

Ellegard, L., Aldenbratt, A., Svensson, M. K., \& Lindberg, C. (2019). Body composition in patients with primary neuromuscular disease assessed by dual energy X-ray absorptiometry (DXA) and three different bioimpedance devices. Clinical Nutrition ESPEN, 29, 142-148. doi:https://doi.org/10.1016/j.clnesp.2018.11.004

Fielding, R. A., Vellas, B., Evans, W. J., Bhasin, S., Morley, J. E., Newman, A. B., . . Zamboni, M. (2011). Sarcopenia: An Undiagnosed Condition in Older Adults. Current Consensus Definition: Prevalence, Etiology, and Consequences. International Working Group on Sarcopenia. Journal of the American Medical Directors Association, 12(4), 249-256. doi:https://doi.org/10.1016/j.jamda.2011.01.003

Gartner, A., Maire, B., Traissac, P., Massamba, J. P., Kameli, Y., Keraudren, V., \& Delpeuch, F. (2000). Sensitivity and specificity of the body mass index to assess low percent body fat in African women. Am J Hum Biol, 12(1), 25-31. doi:10.1002/(sici)15206300(200001/02)12:1<25::aid-ajhb4>3.0.co;2-9

Goncalves, V. S., Faria, E. R., Franceschini Sdo, C., \& Priore, S. E. (2013). Predictive capacity of different bioelectrical impedance analysis devices, with and without protocol, in the evaluation of adolescents. $J$ Pediatr (Rio J), 89(6), 567-574. doi:10.1016/j.jped.2013.03.023

Goodpaster, B. H., Park, S. W., Harris, T. B., Kritchevsky, S. B., Nevitt, M., Schwartz, A. V., . . . Newman, A. B. (2006). The loss of skeletal muscle strength, mass, and quality in older adults: the health, aging and body composition study. The Journals of Gerontology Series A: Biological Sciences and Medical Sciences, 61(10), 1059-1064.

Heymsfield, S. B., Peterson, C. M., Thomas, D. M., Heo, M., \& Schuna, J. M. (2016). Why are there race/ethnic differences in adult body mass index-adiposity relationships? A quantitative critical review. Obesity Reviews, 17(3), 262-275. doi:doi:10.1111/obr.12358

Kabiri, L. S., Hernandez, D. C., \& Mitchell, K. (2015). Reliability, Validity, and Diagnostic Value of a Pediatric Bioelectrical Impedance Analysis Scale. Childhood Obesity, 11(5), 650-655. doi:10.1089/chi.2014.0156

Khalil, S., Mohktar, M., \& Ibrahim, F. (2014). The Theory and Fundamentals of Bioimpedance Analysis in Clinical Status Monitoring and Diagnosis of Diseases. Sensors, 14(6), 10895.

Kim, M., \& Kim, H. (2013). Accuracy of segmental multi-frequency bioelectrical impedance analysis for assessing whole-body and appendicular fat mass and lean soft tissue mass in frail women aged 75 years and older. European Journal Of Clinical Nutrition, 67, 395. doi:10.1038/ejen.2013.9

Kyle, U. G., Genton, L., Hans, D., \& Pichard, C. (2003). Validation of a bioelectrical impedance analysis equation to predict appendicular skeletal muscle mass (ASMM). Clinical Nutrition, 22(6), 537-543. doi:https://doi.org/10.1016/S0261-5614(03)00048-7

Mialich, M. S., Sicchieri, J. F., \& Junior, A. A. J. (2014). Analysis of body composition: a 
critical review of the use of bioelectrical impedance analysis. Int J Clin Nutr, 2(1), 1-10.

Neves, F. S., Leandro, D. A., Silva, F. A., Netto, M. P., Oliveira, R. M., \& Candido, A. P. (2015). Evaluation of the predictive capacity of vertical segmental tetrapolar bioimpedance for excess weight detection in adolescents. J Pediatr (Rio J), 91(6), 551559. doi:10.1016/j.jped.2015.01.006

Okorodudu, D. O., Jumean, M. F., Montori, V. M., Romero-Corral, A., Somers, V. K., Erwin, P. J., \& Lopez-Jimenez, F. (2010). Diagnostic performance of body mass index to identify obesity as defined by body adiposity: a systematic review and meta-analysis. International Journal Of Obesity, 34, 791. doi:10.1038/ijo.2010.5

Pietrobelli, A., Morini, P., Battistini, N., Chiumello, G., Nuñez, C., \& Heymsfield, S. B. (1998). Appendicular skeletal muscle mass: prediction from multiple frequency segmental bioimpedance analysis. European Journal of Clinical Nutrition, 52, 507. doi:10.1038/sj.ejen.1600592

Reiter, R., Iglseder, B., Treschnitzer, W., Alzner, R., Mayr-Pirker, B., Kreutzer, M., . . Reiss, J. (2019). Quantifying appendicular muscle mass in geriatric inpatients: Performance of different single frequency BIA equations in comparison to dual X-ray absorptiometry. Archives of Gerontology and Geriatrics, 80, 98-103. doi:https://doi.org/10.1016/j.archger.2018.10.010

Salinari, S., Bertuzzi, A., Mingrone, G., Capristo, E., Scarfone, A., Greco, A. V., \& Heymsfield, S. B. (2003). Bioimpedance analysis: a useful technique for assessing appendicular lean soft tissue mass and distribution. Journal of Applied Physiology, 94(4), 1552-1556. doi:10.1152/japplphysiol.00571.2002

Sillanpaa, E., Hakkinen, A., \& Hakkinen, K. (2013). Body composition changes by DXA, BIA and skinfolds during exercise training in women. European journal of applied physiology, 113(9), 2331-2341.

Sun, G., French, C. R., Martin, G. R., Younghusband, B., Green, R. C., Xie, Y.-g., . . Zhang, H. (2005). Comparison of multifrequency bioelectrical impedance analysis with dual-energy X-ray absorptiometry for assessment of percentage body fat in a large, healthy population. The American Journal of Clinical Nutrition, 81(1), 74-78. doi:10.1093/ajen/81.1.74

Wang, H., Hai, S., Cao, L., Zhou, J., Liu, P., \& Dong, B.-R. (2016). Estimation of prevalence of sarcopenia by using a new bioelectrical impedance analysis in Chinese communitydwelling elderly people. BMC Geriatrics, 16(1), 216. doi:10.1186/s12877-016-0386-z

Wannamethee, S. G., \& Atkins, J. L. (2015). Muscle loss and obesity: the health implications of sarcopenia and sarcopenic obesity. Proceedings of the Nutrition Society, 74(4), 405-412. doi:10.1017/S002966511500169X 